\title{
Influência dos Stents Farmacológicos na Seleção de Pacientes Diabéticos Tratados por Meio de Intervenção Coronária Percutânea
}

\author{
Luiz Fernando Tanajura', Fausto Feres', Dimytri Alvim Siqueira', Alexandre Abizaid', \\ Suzy Macedo Fraulob', Adriana Fucci', Andréia Mendes Maranhão', Carlos Geraldo Bartolozzi', \\ Silvana Moreira Borges ${ }^{1}$, Danielle Peixoto Marcelino' ${ }^{1}$, Amanda G. M. R. Sousa' ${ }^{1}$, J. Eduardo Sousa ${ }^{1}$
}

\section{RESUMO}

Introdução: Os diabéticos representam um desafio para as intervenções coronárias percutâneas (ICP) em decorrência das elevadas taxas de reestenose, limitando a utilização desse procedimento em situações com grande perspectiva de recidiva, restringindo o emprego da técnica e predispondo à revascularização incompleta. Os stents farmacológicos contribuíram para a atenuação expressiva dessas limitações, podendo ter causado mudanças nas características dos casos tratados contemporaneamente. Assim, nosso objetivo foi comparar o perfil dos diabéticos tratados por ICP em dois momentos distintos desta década. Método: Estudo de coorte envolvendo 2.530 pacientes diabéticos revascularizados de forma consecutiva, divididos em dois grupos: grupo A, 1.309 pacientes revascularizados no período de 2006 a 2008; e grupo B, 1.221 pacientes tratados entre 2003 e 2005. Excluíram-se apenas os pacientes em que foram utilizados stents farmacológicos não disponíveis para uso comercial. Resultados: Os stents farmacológicos foram mais utilizados no grupo $\mathrm{A}$ $(23 \%$ vs. $9 \% ; P<0,0001)$, em que também ocorreu predomínio significante de pacientes em uso de insulina $(14 \%$ vs. $10 \% ; P=0,0001)$, com disfunção renal crônica $(20 \%$ vs. $10,1 \%$; $P<0,0001)$, lesões B2/C (68\% vs. $63 \%$; $P=0,003)$, oclusões crônicas $(17 \%$ vs. $10 \% ; \mathrm{P}<0,0001)$, stents com diâmetro $<2,5 \mathrm{~mm}(26 \%$ vs. $22 \%$; $\mathrm{P}=0,01)$ e stents com extensão $>24 \mathrm{~mm}(31 \%$ vs. $22 \%$; $\mathrm{P}<0,0001)$. Intervenções multiarteriais $(12 \%$ vs. $6 \% ; P<0,001)$ e revascularização completa $(65 \%$ vs. $59 \% ; P=0,002)$ também predominaram no grupo A. Os resultados clínicos hospitalares não diferiram. Conclusão: $\mathrm{A}$ maior disponibilidade de stents farmacológicos causou alterações substanciais no perfil dos diabéticos tratados contemporaneamente, ampliando as indicações para casos mais complexos e proporcionando a obtenção de uma revascularização miocárdica mais completa.

DESCRITORES: Doenças cardiovasculares. Stents farmacológicos. Diabetes mellitus.
ABSTRACT

Influence of Drug-Eluting Stents in the Selection of Diabetic Patients Treated by Percutaneous Coronary Intervention

Background: Diabetic patients represent a challenge for percutaneous coronary interventions $(\mathrm{PCl})$ due to the high restenosis rates, which limit the use of the procedure in cases prone to recurrences, predisposing patients to incomplete revascularization. Drug-eluting stents (DES) have contributed for a significant improvement of these limitations and may have caused changes in the characteristics of patients treated contemporaneously. Thus, our objective was to compare the profile of diabetic patients treated in two different periods of this decade. Method: This was a consecutive cohort study including 2,530 diabetic patients who were divided into two groups: group A, 1,309 patients revascularized from 2006 to 2008; and group B, 1,221 patients treated from 2003 to 2005. Only patients using DES not commercially available were excluded. Results: DES were more frequently used in group A $(23 \%$ vs. $9 \%$; $\mathrm{P}<0.0001$ ), which has a higher prevalence of insulinrequiring patients $(14 \%$ vs. $10 \% ; \mathrm{P}=0.0001)$, chronic renal failure $(20 \%$ vs. $10.1 \% ; \mathrm{P}<0.0001), \mathrm{B} 2 / \mathrm{C}$ lesions $(68 \%$ vs. $63 \% ; \mathrm{P}=0.003)$, total occlusions $(17 \%$ vs. $10 \%$; $\mathrm{P}<0.0001)$, stent diameter $<2.5 \mathrm{~mm}(26 \%$ vs. $22 \%$; $\mathrm{P}=0.01)$ and stent length $>24 \mathrm{~mm}(31 \%$ vs. $22 \%$; $\mathrm{P}<0.0001)$. Multivessel interventions $(12 \%$ vs. $6 \% ; \mathrm{P}<0.001)$ and complete revascularization $(65 \%$ vs. $59 \% ; P=0.002)$ also prevailed in group A. Clinical hospital results were not different between groups. Conclusion: The greater availability of DES has led to significant changes in the profile of diabetic patients treated currently, expanding the indications for more complex patients and providing a more complete myocardial revascularization.

KEY-WORDS: Cardiovascular diseases. Drug-eluting stents. Diabetes mellitus.

1 Instituto Dante Pazzanese de Cardiologia - São Paulo, SP, Brasil. Correspondência: Luiz Fernando Tanajura. Av. Dr. Dante Pazzanese, 500 - Ibirapuera - São Paulo, SP, Brasil - CEP 04012-909 E-mail: Iftanajura@uol.com.br

Recebido em: 15/2/2010 • Aceito em: 2/5/2010 
0 diabetes melito sempre representou um desafio para a cardiologia intervencionista, em razão das altas taxas de eventos cardíacos maiores e reestenose (clínica e angiográfica) apresentadas pelos pacientes tratados. ${ }^{1,2}$ Este fato, na era anterior aos stents com liberação de medicamentos, limitava as indicações da intervenção coronária percutânea para casos de menor complexidade angiográfica ${ }^{1,2}$, menos propensos a recidivas, predispondo também à revascularização incompleta, com suas consequências indesejáveis. ${ }^{3}$

A introdução e o desenvolvimento dos stents farmacológicos mudaram esse cenário de forma radical. ${ }^{4-6} \mathrm{~A}$ eficácia inconteste desses modelos na prevenção da reestenose coronária em virtualmente todos os subgrupos de pacientes possibilitou uma ampliação expressiva das indicações da intervenção coronária percutânea, que passou a ser cogitada em cenários até então proibitivos, pela elevada perspectiva de recidiva das lesões tratadas. Os diabéticos com maior predisposição para o desenvolvimento de reestenose foram alguns dos que mais se beneficiaram dessa nova realidade, o que pode, e deve, ter resultado em mudanças drásticas no perfil dos casos tratados na fase atual, em comparação com situações nas quais esses instrumentos não estavam disponíveis. ${ }^{6,7}$

O objetivo desta investigação foi avaliar a influência do uso mais rotineiro dos stents com liberação de medicamentos nas características clínicas, angiográficas e relacionadas ao procedimento percutâneo em duas séries consecutivas de pacientes diabéticos, tratados em momentos distintos, no tocante à possibilidade de emprego desses modelos.

\section{MÉTODO}

Foram selecionadas para comparação duas populações de pacientes diabéticos dilatados de forma consecutiva em dois períodos distintos: um mais recente (grupo A, tratados entre 2006 e 2008, $\mathrm{n}=1.309$ ), no qual havia maior disponibilidade para a utilização de stents farmacológicos, e outro mais antigo (grupo $B$, tratados entre 2003 e 2005, $n=1.221$ ), no qual a possibilidade de uso desses modelos era mais escassa. Os casos foram identificados a partir de um banco de dados informatizado, no qual foram incluídos de forma prospectiva. A natureza do estudo foi observacional.

Foram excluídos apenas casos tratados por meio de stents farmacológicos ainda não aprovados para uso comercial, já que a utilização de tais modelos está geralmente associada a restrições tanto na seleção dos casos quanto na efetivação do procedimento (situações que se distanciam do chamado mundo real).

É interessante citar a origem dos stents farmacológicos utilizados, na medida em que hospitais públicos, como é o caso do nosso, subvencionado pelo Sistema Único de Saúde, não estão autorizados a im- plantar tais dispositivos de rotina, pelo custo ainda elevado. Em ambas as fases, as fontes foram: 1) participação em ensaios clínicos/registros de mundo real; 2) doações e/ou negociações com as empresas, esta segunda permitindo redução nos custos de aquisição; e 3) casos nos quais o implante era autorizado por seguros-saúde privados.

As intervenções coronárias percutâneas foram realizadas pela técnica convencional de liberação ótima, com implante direto ou pré-dilatação, o que era deixado a critério do cardiologista intervencionista. A farmacologia adjunta consistiu de: 1) heparina nãofracionada, na dose de $100 \mathrm{UI} / \mathrm{kg}$ peso, administrada imediatamente antes do procedimento, com o objetivo de atingir tempo de coagulação ativada igual ou superior a 250 segundos; 2) aspirina (dose de ataque de $300 \mathrm{mg}$, seguida de $100 \mathrm{mg} / \mathrm{dia}$ ), iniciada pelo menos na véspera da intervenção e mantida indefinidamente; 3) clopidogrel (dose de ataque de $300 \mathrm{mg}$, iniciada pelo menos 24 horas antes da intervenção, seguida por $75 \mathrm{mg} / \mathrm{dia}$ ) ou ticlopidina (500 mg/dia, com início 72 horas antes da intervenção coronária percutânea, mantendo-se a mesma dose pelo tempo recomendado) - em situações de intervenção coronária percutânea não planejada, era utilizada dose de $600 \mathrm{mg}$ de clopidogrel, no mínimo duas horas antes da intervenção coronária percutânea (o esquema antiplaquetário duplo era mantido, no período de 2003 a 2006, por três a seis meses, e, a partir de 2007, por pelo menos um ano após o uso de um stent farmacológico); e 4) inibidores da glicoproteína Illb/llla foram utilizados nas doses habituais, quando indicados ou preconizados pelos protocolos dos estudos clínicos.

Para que as intervenções coronárias percutâneas fossem indicadas, deveria haver isquemia miocárdica espontânea (angina do peito ou infarto) ou induzida (positividade dos testes funcionais) e presença de pelo menos uma lesão com diâmetro de estenose igual ou superior a $70 \%$ pelo critério visual, adequada para dilatação. As lesões-alvo foram classificadas de acordo com a proposta da Força-Tarefa Americana. ${ }^{8}$ As artérias tratadas foram discriminadas em troncos principais (descendente anterior, coronária direita e circunflexa) e ramos secundários dos mesmos. A função ventricular esquerda foi avaliada pela análise visual da fração de ejeção do ventrículo esquerdo, na projeção OAD, considerando-se comprometimento moderado ou grave achado inferior a $45 \%$. Angiografia coronária quantitativa e ultrassom intracoronário não foram realizados de rotina.

Considerou-se sucesso do procedimento a obtenção de estenose residual inferior a $20 \%$ pelo critério visual, na ausência de complicações maiores (óbito, infarto do miocárdio ou cirurgia de urgência) na fase hospitalar. Infarto do miocárdio foi caracterizado por elevações da creatina quinase fração MB (CK-MB) superiores a três vezes o valor normal. 
A comparação entre as variáveis quantitativas foi feita utilizando-se o teste $t$ de Student. Para as comparações referentes às variáveis qualitativas utilizou-se o teste de qui-quadrado. Consideraram-se significantes valores de $\mathrm{P}<0,05$.

\section{RESULTADOS}

No período de 2006 a 2008, 1.309 diabéticos (29,3\% dos 4.454 pacientes revascularizados) foram tratados e comparados a 1.221 diabéticos $(29,2 \%$ dos 4.174 revascularizados) submetidos a intervenção coronária percutânea no período de 2003 a 2005 ( $P=0,45)$. Observamos expressivo aumento do emprego de stents farmacológicos na fase mais contemporânea $(23,3 \%$ vs. $9,4 \% ; P<0,0001)$.

A Tabela 1 discrimina as principais características clínicas, que exibiram claras diferenças entre os grupos, enfatizando-se: predomínio significativo de casos em uso de insulina $(14,4 \%$ vs. 9,9\%; $P=0,0001)$, disfunção renal crônica (20,2\% vs. 10,1\%; P < 0,0001) e antecedente de infarto do miocárdio no grupo $A$ $(42,0 \%$ vs. $38 \% ; P=0,04)$. As formas clínicas de apresentação da doença coronária não diferiram.

Em relação à terapêutica clínica adjunta antitrombótica, os inibidores da glicoproteína IIb/IIla (5,7\% vs. $2,9 \% ; P=0,0009)$ e o clopidogrel $(39,5 \%$ vs. $27,1 \%$; $\mathrm{P}<0,0001)$ foram mais frequentemente administrados nos casos do grupo A.

Dentre os dados associados à angiografia coronária de base, observou-se doença multiarterial em 40,5\% dos pacientes do grupo A e em 35,7\% dos casos do grpo $B(P=0,01)$. As demais características encontram-se discriminadas na Tabela 2, destacando-se predomínio expressivo de intervenções em lesões-alvo de maior complexidade, inclusive oclusões crônicas (17\% vs. 10,4\%; P = 0,0001), no grupo A. Nesse grupo também foram observados mais casos com disfunção ventricular esquerda significante (19,2\% vs. 12\%; $P<0,0001)$. A distribuição dos vasos tratados também foi distinta, possivelmente pela presença de mais lesões situadas em ramos secundários no grupo $A$.

No tocante às variáveis do procedimento, ocorreram maiores porcentuais de revascularização completa $(65,6 \%$ vs. 59,6\%; P = 0,002) e intervenção coronária percutânea de múltiplos vasos (12,4\% vs. 6,7\%; $\mathrm{P}<0,0001)$ nos pacientes tratados mais contemporaneamente. A Figura 1 compara a extensão e o diâmetro dos modelos implantados, observando-se que nos casos do grupo A foram utilizados, em média, stents mais longos e de menor diâmetro.

Por fim, a Tabela 3 ilustra os principais resultados clínicos na fase hospitalar, os quais não diferiram entre os grupos, com porcentuais de óbito, infarto e cirurgia de urgência sempre abaixo de $1 \%$.

\section{DISCUSSÃO}

O diabetes melito é uma afeccção das mais comuns na prática clínica, tendo grande potencial de comprometer a qualidade (recidiva dos sintomas em casos de reestenose ou revascularização incompleta) e

TABELA 1

Características clínicas

\begin{tabular}{|c|c|c|c|}
\hline Variáveis & $\begin{array}{c}\text { Grupo A } \\
(n=1.309)\end{array}$ & $\begin{array}{c}\text { Grupo B } \\
(n=1.221)\end{array}$ & $\mathbf{P}$ \\
\hline Média de idade & $64,3 \pm 10,7$ anos & $62,0 \pm 10,1$ anos & $<0,0001$ \\
\hline Sexo masculino & $59,8 \%$ & $61,5 \%$ & 0,40 \\
\hline História de tabagismo & $36,9 \%$ & $51,9 \%$ & $<0,0001$ \\
\hline Diabetes em uso de insulina & $14,5 \%$ & $9,9 \%$ & 0,0001 \\
\hline Hipertensão arterial sistêmica & $92,6 \%$ & $87,2 \%$ & $<0,0001$ \\
\hline Hipercolesterolemia & $75 \%$ & $73,9 \%$ & 0,57 \\
\hline Insuficiência renal & $20,2 \%$ & $10,1 \%$ & $<0,0001$ \\
\hline Infarto prévio & $42 \%$ & $38 \%$ & 0,04 \\
\hline RM prévia & $14,6 \%$ & $16,2 \%$ & 0,28 \\
\hline Intervenção percutânea prévia & $22,6 \%$ & $24,5 \%$ & 0,03 \\
\hline Apresentação clínica & & & 0,41 \\
\hline Angina estável & $44 \%$ & $43,8 \%$ & \\
\hline Isquemia silenciosa & $28,4 \%$ & $29,8 \%$ & \\
\hline SCA sem SST & $19,2 \%$ & $19,7 \%$ & \\
\hline IAM com SST & $8,4 \%$ & $6,7 \%$ & \\
\hline
\end{tabular}

IAM = infarto agudo do miocárdio; $\mathrm{n}$ = número de pacientes; $\mathrm{RM}=$ revascularização miocárdica cirúrgica; $\mathrm{SCA}=$ síndromes coronárias agudas; SST = supradesnivelamento do segmento ST. 
TABELA 2

Características da angiografia pré-procedimento

\begin{tabular}{lccc}
\hline Variáveis & $\begin{array}{c}\text { Grupo A } \\
(\mathbf{n}=\mathbf{1 . 3 0 9})\end{array}$ & $\begin{array}{c}\text { Grupo B } \\
(\mathbf{n = 1 . 2 2 1})\end{array}$ & $\mathbf{P}$ \\
\hline Vasos tratados & & & $<0,0001$ \\
$\quad$ Descendente anterior & $33,4 \%$ & $35,6 \%$ & \\
Coronária direita & $30,8 \%$ & $28,2 \%$ & \\
$\quad$ Circunflexa & $19,2 \%$ & $20,9 \%$ & \\
Ramos secundários & $13 \%$ & $9,1 \%$ & 0,003 \\
$\quad$ Enxertos venosos & $3,6 \%$ & $4,2 \%$ & 0,15 \\
Tipo de lesão & & & 0,38 \\
$\quad$ B2/C & $68,6 \%$ & $63,4 \%$ & 0,002 \\
Lesões reestenóticas & $6,9 \%$ & $5,4 \%$ & 0,0001 \\
Lesões em bifurcações & $5,7 \%$ & $5 \%$ & $<0,0001$ \\
Localização medial/distal & $38,3 \%$ & $32,7 \%$ & $10,4 \%$ \\
Oclusões crônicas & $17 \%$ & $12 \%$ & \\
Disfunção do VE moderada/grave & $19,2 \%$ & & \\
\hline
\end{tabular}

$\mathrm{n}$ = número de pacientes; VE = ventrículo esquerdo.

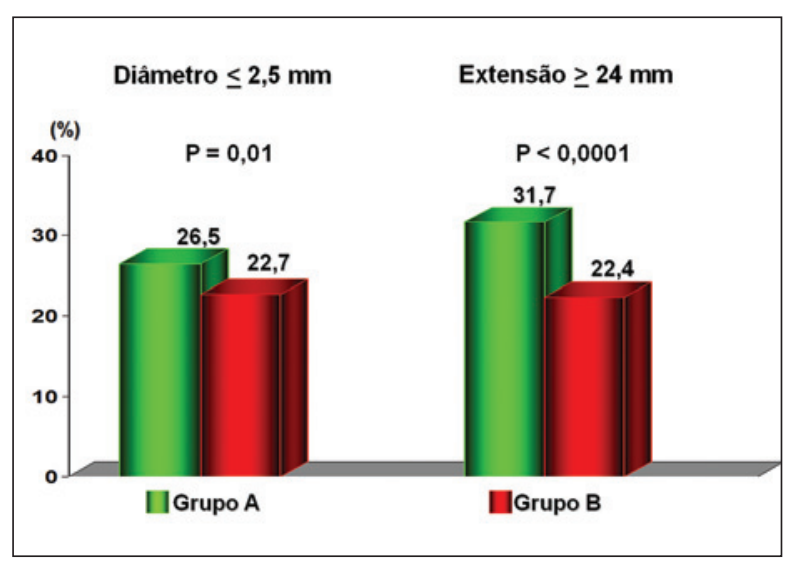

Figura 1 - Diâmetro e extensão dos stents implantados.

a quantidade de vida (doença associada a aumento significativo de infarto do miocárdio e mortalidade de causa cardiovascular na fase tardia) de seus portadores. ${ }^{9-11}$ Estima-se que, em alguns anos, o número de pacientes acometidos ultrapassará os 350 milhões. Especificamente no que tange às intervenções coronárias percutâneas, essa doença é classicamente considerada preditor independente de reestenose e de revascularização incompleta. ${ }^{9-11}$

O desenvolvimento e o uso clínico dos stents farmacológicos acompanharam-se de reduções acentuadas nos porcentuais de reestenose angiográfica/clínica, com reflexo nas revascularizações adicionais no primeiro ano após a dilatação, o que foi observado em praticamente todos os subgrupos tratados com esses dispositivos, independentemente do modelo emprega- do. ${ }^{6,12,13}$ Em consequência, casos com alta predisposição para reestenose, mesmo que ainda não devidamente avaliados por meio de ensaios clínicos específicos (indicações off-label), passaram a ser tratados de forma rotineira, ampliando o espectro das intervenções coronárias percutâneas e possibilitando maior obtenção de revascularização miocárdica completa, sempre almejada. ${ }^{3,12,13}$ Mais recentemente, essas afirmações ganharam o respaldo de ensaios clínicos, que ratificaram a segurança desses modelos, inclusive com redução da mortalidade. ${ }^{14,15}$

Os diabéticos foram alguns dos que mais se beneficiaram com esse avanço, conforme demonstrou o estudo DIABETES ${ }^{16}$, no qual os stents farmacológicos com liberação de sirolimus apresentaram significante vantagem sobre os modelos não revestidos nas variáveis perda tardia da luz $(0,06 \mathrm{~mm}$ vs. 0,47 mm; $P<0,001)$, reflexo indireto da hiperplasia neointimal, cerne das recidivas, bem como nas reestenoses intrastent $(2,7 \%$ vs. $27,5 \% ; \mathrm{P}<0,001)$, com consequente redução expressiva dos eventos cardíacos maiores em um ano (11,3\% vs. 36.3\%; P<0,001). Análises de subgrupos demonstraram vantagem do stent farmacológico em praticamente todas as principais variáveis clínicas e angiográficas. Esses resultados foram considerados tão consistentes que ensaios clínicos posteriores nos diabéticos passaram a comparar diferentes modelos de stents farmacológicos e não mais próteses revestidas e não-revestidas. ${ }^{17}$

Em nosso meio, os pacientes tratados em hospitais públicos de grande movimento, como o nosso, não têm acesso a essa tecnologia, pelo fato de o Sistema único de Saúde, fonte predominante de custeio das intervenções 
Tanajura LF, et al. Influência dos Stents Farmacológicos na Seleção de Pacientes Diabéticos Tratados por Meio de Intervenção Coronária Percutânea. Rev Bras Cardiol Invasiva. 2010;18(2):151-6.

TABELA 3

Resultados clínicos na fase hospitalar

\begin{tabular}{lccc}
\hline Variáveis & $\begin{array}{c}\text { Grupo A } \\
(\mathbf{n = 1 . 3 0 9 )}\end{array}$ & $\begin{array}{c}\text { Grupo B } \\
(\mathbf{n = 1 . 2 2 1})\end{array}$ & $\mathbf{P}$ \\
\hline Sucesso do procedimento & $97,1 \%$ & $97,9 \%$ & 0,25 \\
Complicações maiores & $0,9 \%$ & $0,5 \%$ & 0,24 \\
Óbito & $0,8 \%$ & $0,4 \%$ & 0,21 \\
Infarto do miocárdio & $0,3 \%$ & $0,08 \%$ & 0,37 \\
Cirurgia de emergência & 0 & 0 & NA \\
\hline
\end{tabular}

$\mathrm{n}=$ número de pacientes; NA = não aplicável.

coronárias percutâneas, não autorizar o implante desses dispositivos, pelo alto custo. ${ }^{6}$ No entanto, como já comentado, fruto da participação em ensaios clínicos e registros envolvendo casos do mundo real, bem como por doações e negociações com fornecedores, logramos aumento progressivo do emprego desses modelos, cuja utilização passou de 9,4\% no triênio 2003-2005 para 23,3\% no período 2006-2008 ( $P<0,0001)$.

Os resultados dessa investigação, que abrangeu 2.530 pacientes diabéticos consecutivamente dilatados, demonstraram que essa maior disponibilidade de stents farmacológicos contribuiu para que pudéssemos tratar no momento atual casos bem mais predispostos ao desenvolvimento de reestenose, mais especificamente os pacientes dependentes de insulina, os portadores de disfunção renal crônica, os que exibiam estenoses segmentares em vasos de fino calibre, muitas localizadas nas porções distais das artérias, assim como as oclusões coronárias crônicas. , $^{6,12-15}$ Além disso, os pacientes do grupo A também eram de maior gravidade clínica, o que foi demonstrado pelo predomínio significante de casos com acometimento de múltiplos vasos e maior porcentual de disfunção ventricular de grau moderado ou grave.

Casos dependentes de insulina são fortes predisponentes de eventos pós-intervenção coronária percutânea, e não apenas reestenose e as reintervenções indicadas para seu tratamento, mas também trombose coronária e morte, o que é agravado sobremaneira pela presença de disfunção renal concomitante, conforme demonstraram os achados do registro e-Cypher. ${ }^{18}$ O mesmo ocorre nos que evidenciam acometimento de vasos de menor diâmetro de referência, em especial se apresentarem estenoses segmentares, também associados a maiores taxas de reobstrução pelo critério binário. ${ }^{7,13}$ Aparentemente, essa predisposição decorre do fato de os volumes de hiperplasia neointimal não dependerem do diâmetro da artéria-alvo, facilitando o desenvolvimento de reestenose nos vasos mais finos, o que é agravado de forma clara e consistente pelo diabetes. ${ }^{7,13} \mathrm{O}$ benefício do implante de stents farmacológicos nesses casos também já está claramente demonstrado ${ }^{7,13-15}$, igualmente explicando o achado desta investigação, na qual os pacientes do grupo A receberam mais stents longos (extensão superior a $24 \mathrm{~mm}$ ) e implantados em vasos de menor diâmetro (inferior a 2,5 mm). O predomínio de lesões localizadas nas porções média ou distal das estenoses nos pacientes tratados mais contemporaneamente (grupo A), além do provável predomínio de intervenção coronária percutânea em lesões envolvendo ramos secundários, também nos pareceu uma evidência robusta de estarmos tratando mais vasos de fino calibre na atualidade.

A oportunidade de tratar de forma mais efetiva casos com alta predisposição para as recidivas redundou em maior porcentual de intervenção coronária percutânea de múltiplos vasos e, consequentemente, maior obtenção de revascularização completa no grupo A, a qual é sempre preferível por acompanhar-se de melhores resultados na evolução clínica tardia. ${ }^{3}$ Deve ainda ser mencionado que a intervenção em variedades anatômicas de maior complexidade não foi acompanhada de piora dos resultados clínicos na fase hospitalar, contrariando os achados do registro EVENT ${ }^{19}$, no qual as dilatações nas chamadas situações off-label resultaram em aumento significativo dos eventos cardíacos maiores durante a hospitalização inicial $(11 \%$ vs. $5 \%$; P $<0,001)$.

As mudanças observadas na farmacologia adjunta antitrombótica também devem ser destacadas. O aumento da prescrição do clopidogrel, medicamento mais seguro e com maior facilidade de administração que a ticlopidina, nos pareceu ser um reflexo direto da queda dos preços desse fármaco. Já a maior utilização dos inibidores IIb/IIla pode ser explicada de duas formas: 1) recomendação explícita de protocolos de ensaios clínicos, como é o caso do estudo FREEDOM, motivada pela crença de que esses fármacos poderiam reduzir a reestenose nos diabéticos ${ }^{20}$, hipótese formulada em meados dos anos 90, após os resultados do ensaio clínico EPISTENT; e 2) tratamento de lesões de maior complexidade. É imperativo citar que nos dias atuais a indicação mais comum para os inibidores Ilb/IIla são as intervenções coronárias percutâneas primárias no infarto, forma de apresentação que não diferiu entre os grupos. 
Finalizando, as demais diferenças observadas entre os grupos A e B nos pareceram muito mais decorrentes do caráter retrospectivo do estudo e de casualidades que de reais diferenças clínicas entre os pacientes tratados. Um exemplo claro dessa assertiva foi o predomínio de antecedentes de intervenção coronária percutânea no grupo $B$, sem que esse achado fosse acompanhado de mais dilatações para o tratamento de lesões reestenóticas, como seria de se esperar. Talvez a única exceção seja a redução do número de tabagistas na fase mais contemporânea, o que reflete a grande ênfase atual nas medidas de prevenção secundária. ${ }^{6}$

\section{Limitações}

As seguintes limitações devem ser comentadas: 1) a investigação não foi prospectiva, não havendo quaisquer análises preestabelecidas quando da inclusão dos casos no banco de dados; 2) não foi realizada angiografia quantitativa nos pacientes, impossibilitando uma comparação efetiva de algumas características relevantes das lesões tratadas; e 3) a ausência de evolução clínica tardia impediu que se pudesse constatar benefício clínico, possivelmente de grande expressão, oriundo da maior utilização de stents farmacológicos.

\section{CONCLUSÃO}

A maior disponibilidade da utilização de stents farmacológicos nos últimos anos causou sensíveis mudanças no perfil dos pacientes diabéticos tratados, possibilitando a abordagem de casos bem mais propensos ao desenvolvimento de reestenose, muitos dos quais poderiam ter sido recusados para dilatação em situações nas quais esses instrumentos não estivessem disponíveis. Além disso, também ocorreu significativo aumento de revascularização miocárdica completa, desejável na maior parte das situações.

\section{CONFLITO DE INTERESSES}

Os autores declararam inexistência de conflito de interesses relacionado a este manuscrito.

\section{REFERÊNCIAS}

1. Elezi S, Kastrati A, Pache J, Wehinger A, Hadamitzky M, Dirschinger J, et al. Diabetes mellitus and the clinical and angiographic outcome after coronary stent placement. J Am Coll Cardiol. 1998;32(7):1866-73.

2. Abizaid A, Kornowski R, Mintz GS, Hong MK, Abizaid AS, Mehran R, et al. The influence of diabetes mellitus on acute and late clinical outcomes following coronary stent implantation. J Am Coll Cardiol. 1998;32(3):584-9.

3. Hannan EL, Wu C, Walford G, Holmes DR, Jones RH, Sharma S, et al. Incomplete revascularization in the era of drug-eluting stents: impact on clinical outcomes. JACC Cardiovasc Interv. 2009;2(1):17-25.

4. Sousa JE, Costa MA, Abizaid A, Abizaid AS, Feres F, Pinto $I M$, et al. Lack of neointimal proliferation after implantation of sirolimus-coated stents in human coronary arteries: a quantitative coronary angiography and three-dimensional intravascular ultrasound study. Circulation. 2001;103(2):192-95.
5. Ong AT, Serruys PW, Aoki J, Hoye A, van Mieghem CA, Rodriguez-Granillo GA, et al. The unrestricted use of paclitaxelversus sirolimus-eluting stents for coronary artery disease in an unselected population: one-year results of the Taxus-Stent Evaluated at Rotterdam Cardiology Hospital (T-SEARCH) registry. J Am Coll Cardiol. 2005;45(7):1135-41.

6. Tanajura LFL, Feres F, Abizaid AC, Mattos LAP, Siqueira DA, Centemero MP, et al. Stents farmacológicos no mundo real: impacto de sua disponibilidade no perfil de pacientes tratados por meio de intervenção coronária percutânea em um hospital público. Rev Bras Cardiol Invasiva. 2008;16(1):31-6.

7. Yang TH, Park SW, Hong MK, Park DW, Park KM, Kim YH, et al. Impact of diabetes mellitus on angiographic and clinical outcomes in the drug-eluting stents era. Am J Cardiol. 2005; 96(10):1389-92.

8. Smith SC, Dove JT, Jacobs AK, Ward Kennedy J, Kereiakes D, Kern MJ, et al. ACC/AHA guidelines for percutaneous coronary intervention (Revision of the 1993 PTCA guidelines) - Executive summary: a report of the American College of Cardiology/American Heart Association task force on practice guidelines. Circulation. 2001;103(24):3019-41.

9. Leal J, Gray AM, Clarke PM. Development of life-expectancy tables for people with type 2 diabetes. Eur Heart J. 2009;30: 834-9.

10. Franco OH, Steyerberg EW, Hu FB, Mackenbach J, Nusselder W. Association of diabetes mellitus with total life expectancy and life expectancy with and without cardiovascular disease. Arch Intern Med. 2007;167:1145-51.

11. Banning AP, Westaby S, Morice MC, Kappetein AP, Mohr FW, Berti $S$, et al. Diabetic and nondiabetic patients with left main and/or 3-vessel coronary artery disease: comparison of outcomes with cardiac surgery and paclitaxel-eluting stents. J Am Coll Cardiol. 2010;55(11):1067-75.

12. Beohar N, Davidson CJ, Kip KE, Goodreau L, Vlachos HA, Meyers SN, et al. Outcomes and complications associated with off-label and untested use of drug- eluting stents. JAMA. 2007;297:1992-2000.

13. Colombo AC, Chieffo A. Drug-eluting stent update 2007. Part III: technique and unapproved/unsettled indications. Circulation. 2007;116:1424-32.

14. Tu JV, Bowen J, Chiu M, Ko DT, Austin PC, He Y, et al. Effectiveness and safety of drug-eluting stents in Ontario. $\mathrm{N}$ Engl J Med. 2007;357(14):1393-402.

15. Kirtane AJ, Gupta A, lyengar S, Moses JW, Leon MB, Applegate $R$, et al. Safety and efficacy of drug-eluting and bare metal stents. Circulation. 2009;119(25):3198-206.

16. Sabaté M, Jiménez-Quevedo P, Angiolillo DJ, Gómez-Hospital JA, Alfonso F, Goicolea J, et al Randomized comparison of sirolimus-eluting stent versus standard stent for percutaneous coronary revascularization in diabetic patients. The diabetes and sirolimus-eluting stent (DIABETES) trial. Circulation. 2005; 112:2175-83.

17. Dibra A, Kastrati A, Mehilli J, Pache J, Schühlen H, von Beckerath $\mathrm{N}$, et al. Paclitaxel-eluting or sirolimus-eluting stents to prevent restenosis in diabetic patients. N Engl J Med. 2005; 353(7):663-70.

18. Urban P, Gershlick AH, Guagliumi G, Guyon P, Lotan C, Schofer J, et al. Safety of coronary sirolimus-eluting stents in daily clinical practice: one-year follow-up of the e-Cypher registry. Circulation. 2006;113(11):1434-1.

19. Win HK, Caldera AE, Maresh K, Lopez J, Rihal CS, Parikh MA, et al. Clinical outcomes and stent thrombosis following offlabel use of drug-eluting stents. JAMA. 2007;297(18):2001-9.

20. Ibbotson T, McGavin JK, Goa KL. Abciximab: an updated review of this therapeutic use in patients with ischaemic heart disease undergoing percutaneous coronary revascularization. Drugs. 2003;63(11):1121-63. 